\title{
Giant Photoresponsivity of Midinfrared Hyperbolic Metamaterials in the Photon-Assisted-Tunneling Regime
}

\begin{abstract}
Pai-Yen Chen, ${ }^{1,}{ }^{*}$ Mehdi Hajizadegan, ${ }^{1}$ Maryam Sakhdari, ${ }^{1}$ and Andrea Alù ${ }^{2}$
${ }^{1}$ Department of Electrical and Computer Engineering, Wayne State University, Detroit, Michigan 48202, USA

${ }^{2}$ Department of Electrical and Computer Engineering, University of Texas at Austin, Austin, Texas 78712, USA

(Received 22 September 2015; revised manuscript received 20 November 2015; published 28 April 2016)

We explore broadband and omnidirectional midinfrared rectification based on nanopatterned hyperbolic metamaterials, composed of two dissimilar metals separated by an ultrathin dielectric layer. The exotic slow-light modes supported by such periodically trenched hyperbolic metamaterials efficiently trap incident radiation in massively parallel metal-insulator-metal tunnel junctions producing ultrafast infrared rectification via photon-assisted tunneling. This leads to a highly efficient photon-to-electron transduction with obtained photocurrent orders of magnitude larger than conventional lumped-element devices, such as nanorectennas and point-contact rectifiers. Our results promise an impact on infrared energy harvesters and plasmonic photodetectors.
\end{abstract}

DOI: 10.1103/PhysRevApplied.5.041001

Photon-assisted tunneling is an intrinsic quantum-size effect in plasmonic nanostructures, which has recently attracted growing interest because of its exotic nonlinear and nonlocal optical properties [1-6]. The excitation of surface plasmon polariton resonances, combined with tunneling-induced optical nonlinearities, may allow a rich variety of nonlinear optical phenomena, such as efficient harmonic generation, multiwave mixing, resistive switching, and two-photon absorption [7-13]. The second-order nonlinear phenomenon of optical rectification is of particular interest, since it may find potential applications in photodetection and photovoltaics at midinfrared (MIR) wavelengths $[14,15]$. In the MIR regime, a major thrust of renewable-energy research contains harvesting the roughly $10^{17} \mathrm{~W}$ of infrared thermal radiation that Earth continuously emits into outer space [16]. Nonetheless, there exists no suitable MIR-band-gap semiconductors $[16,17]$ that can make an efficient interband photodetector or photovoltaic device, due to the frequent Auger recombination and generation at room temperature. Arguably, rectifying antennas (or "rectennas"), which have been commonly used to generate power from microwaves with a $>90 \%$ power conversion efficiency [18], may realize emissive energy harvesters [14-16]. In this context, a nanorectenna operated at infrared wavelengths can collect infrared radiation and, when loaded with a metal-insulatormetal (MIM) tunneling diode, can convert light into electricity through nonlinear optical rectification [14-16]. The ultrashort tunneling time $\left(\sim 10^{-15} \mathrm{~s}\right)$ in a MIM tunneling diode, working at room temperature, suggests an intrinsic cutoff frequency up to several hundreds of $\mathrm{THz}$ and a high operating speed [19]. However, to date,

*pychen@wayne.edu proof-of-principle nanorectennas have shown insufficient responsivity $\gamma$ at low bias voltages (less than $\mathrm{mA} / \mathrm{W}$ ) [20-22], far from practical use in photodetection $(\sim 0.1 \mathrm{~A} / \mathrm{W})$ and photovoltaics. The major roadblock toward high-performance and viable infrared rectennas stems from inefficient light coupling, yielded by a tradeoff between maximum parasitic cutoff frequency and device dimensions, i.e., diode area and insulator (tunnel barrier) thickness [14,21], an issue that remains unsolved in conventional antenna-diode architectures.

In this Letter, we explore the possibility of using a metamaterial approach, namely hyperbolic metamaterials (HMMs) formed by parallel arrays of asymmetric MIM tunnel junctions, to realize highly efficient midinfrared rectification, as shown in Fig. 1(a). In this multilayered HMM structure, the effective permittivity tensor is $\overline{\bar{\varepsilon}}_{\text {eff }}=\varepsilon_{0}\left(\varepsilon_{\|} \hat{\mathbf{x}} \hat{\mathbf{x}}+\varepsilon_{\|} \hat{\mathbf{y}} \hat{\mathbf{y}}+\varepsilon_{\perp} \hat{\mathbf{z}} \hat{\mathbf{z}}\right) \quad$ [23-25], where $\varepsilon_{\|}=$ $\sum_{i=1}^{N} \varepsilon_{i} \rho_{i}$ and $\varepsilon_{\perp}=\left[\sum_{i=1}^{N} \varepsilon_{i}^{-1} \rho_{i}\right]^{-1}, \varepsilon_{i}$, and $\rho_{i}$ are relative permittivity and volume fraction of the $i$ th constituent materials, respectively. A multilayered HMM can behave as a uniaxially anisotropic medium [23-25], provided that extreme material properties $\operatorname{Re}\left[\varepsilon_{\perp}\right] \operatorname{Re}\left[\varepsilon_{\|}\right]<0$ can be supported such that the isofrequency surfaces become open hyperboloids, as illustrated in the inset of Fig. 1(a).

So far, HMMs have been demonstrated to exhibit negative refraction, hyperlensing, Dyakonov plasmons, and anomalously large photonic density of states (PDOS) in a broad spectral range [23-25]. In this context, HMMs may represent an exciting platform, which holds the greatest promise for developing nanophotonic and quantum-optical devices, such as nonresonant single-photon sources [26] and substrates that can mold spontaneous emission into directional beams [27]. Moreover, the use of a nanostructured HMM substrate (e.g., tapered HMM waveguide array [28-31]) may realize "rainbow trapping," with a broadband 


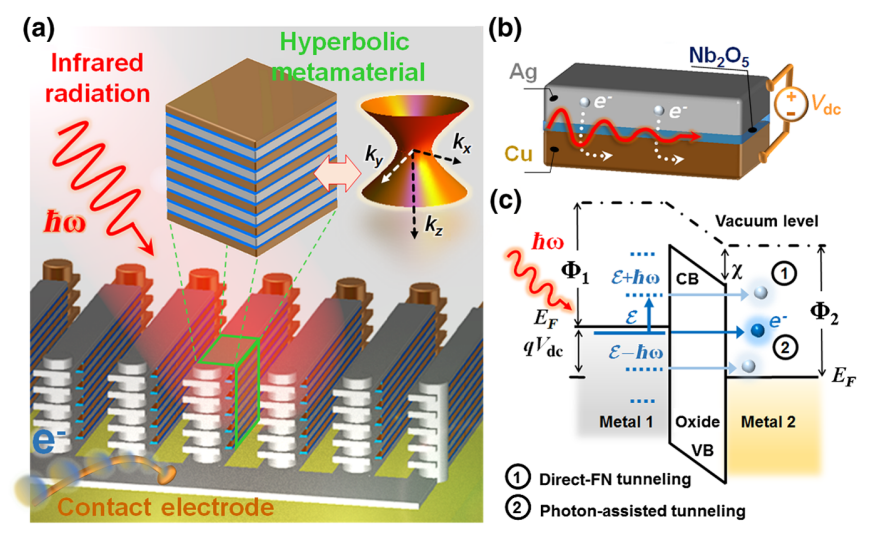

FIG. 1. Schematics of (a) an infrared rectifying device composed of nanopatterned HMMs, (b) nonlinear optical rectification in $\mathrm{Ag}-\mathrm{Nb}_{2} \mathrm{O}_{5}-\mathrm{Cu}$ tunnel junction forming the HMM, (c) energyband diagram of photon-assisted tunneling, (static) direct tunneling, and Fowler-Nordheim (FN) tunneling in a dissimilar MIM tunnel junction; $\mathrm{CB}$ and $\mathrm{VB}$ represent the conduction and valance bands of the insulator.

omnidirectional optical absorption, due to the superposition of multiple slow-wave modes. Beyond these concepts, we propose here an application of HMM substrates in the realization of highly efficient infrared rectification and photodetection. When a HMM unit cell consists of two different metals and two ultrathin, wide-band-gap, and high-electron-affinity insulators of the same kind (e.g., transition-metal oxides) [Fig. 1(b)], effectively forming asymmetric MIM nanodiodes, the HMM may operate in the quantum regime, based on which nonlinear photonassisted-tunneling transport leads to a set of linear and nonlinear quantum conductivities $[12,13]$. By nanopattering this quantum metamaterial as an effective slow-light medium [28-31], a rainbow-trapping effect may allow scattering light into plasmonic nanogaps (i.e., MIM tunneling nanojunctions). In addition, the strong confinement of optical fields will substantially enhance nonlinear optical processes, such as the optical rectification associated with the photocurrent generation. In this scenario, a high infrared responsivity may be obtained by rectifying slow light in the HMM-based traveling-wave structure, somehow similar to traveling-wave microwave and optoelectronic devices [32].

In the following, we theoretically investigate the performance of the proposed HMM-based infrared rectifying devices, considering the interaction of electromagnetic radiation and nonlinear quantum transport. Figure 1(c) illustrates the photon-assistedtunneling and static-tunneling processes in a MIM tunnel junction: in a general scenario, the voltage across the MIM junction can be assumed to be a combination of optical and dc components $V(t)=V_{\mathrm{dc}}+(1 / 2)\left(V_{\omega} e^{-i \omega t}+\right.$ c.c. $)$. From the Tien-Gordon theory $[12,13]$, the new timedependent wave function for electrons in metal becomes $\quad \psi(\overline{\mathbf{r}}, t)=\psi_{0}(\overline{\mathbf{r}}, t) \exp \left[-i / \hbar \int{ }^{t} q V\left(t^{\prime}\right) d t^{\prime}\right]=$ $\psi_{0}(\overline{\mathbf{r}}, t) \sum_{n=-\infty}^{n=+\infty} J_{n}\left(q V_{\omega} / \hbar \omega\right) e^{-i n \omega t}$, where $q$ is the electron charge, $\hbar$ is the reduced Planck constant, $\psi_{0}(\overrightarrow{\mathbf{r}}, t)$ is the unperturbed Schrödinger wave function $[12,13]$, and $J_{n}(\cdot)$ is the $n$ th-order Bessel function of the first kind. The modified wave function implies that optical signals adiabatically modulate the electron potential energy. The monochromatic electromagnetic field may therefore excite new quantum-well virtual states separated from the unperturbed ground state by $\pm n \hbar \omega$, where $n$ corresponds to the number of photons absorbed or emitted, with a probability $J_{n}^{2}\left(q V_{\omega} / \hbar \omega\right)$, by an electron on the metal surface. As a result, the time-dependent current density is in the form of a Fourier series $\underline{J}(t)=\sum_{m=0}^{m=+\infty} \frac{1}{2}\left(\underline{J}_{m \omega} e^{-i m \omega t}+\right.$ c.c. $)$. The $\mathrm{dc}(m=0)$ and $m$ th frequency-dependent currents under illumination are given by

$$
\begin{aligned}
\underline{J}_{\mathrm{dc}}= & \sum_{n=-\infty}^{\infty} J_{0}^{2}\left(q V_{\omega} / \hbar \omega\right) \underline{J}_{\mathrm{dark}}\left(q V_{\mathrm{dc}}+n \hbar \omega\right), \\
\underline{J}_{m \omega}= & \sum_{n=-\infty}^{\infty} J_{n}\left(q V_{\omega} / \hbar \omega\right)\left[J_{n+m}\left(q V_{\omega} / \hbar \omega\right)\right. \\
& \left.+J_{n-m}\left(q V_{\omega} / \hbar \omega\right)\right] \underline{J}_{\mathrm{dark}}\left(q V_{\mathrm{dc}}+n \hbar \omega\right),
\end{aligned}
$$

where the static-tunneling current (dark current) $\underline{J}_{\text {dark }}$ flowing between two dissimilar metals can be calculated using the well-known Simmons' formula [33-35]. At infrared and visible wavelengths where $q V_{\omega} / \hbar \omega \ll 1$, the summation in Eq. (1) can be approximated by terms up to first order $(n=0, \pm 1)$. In this case, the relations between current densities and electric field inside the MIM gap $E_{\omega}\left(V_{\omega}=\int_{0}^{d} E_{\omega} d z\right)$ are derived as $\underline{J}_{\mathrm{dc}} \approx \underline{J}_{\mathrm{dark}}\left(V_{\mathrm{dc}}\right)+$ $\sigma_{0}^{(2)}\left|E_{\omega}\right|^{2}$ and $\underline{J}_{\omega} \approx \sigma_{\omega}^{(1)} E_{\omega}$, where the linear conductivity $\sigma_{\omega}^{(1)}$ and second-order quantum conductance $\sigma_{0}^{(2)}$, responsible for the optical rectification [13], are given by

$$
\begin{aligned}
& \sigma_{\omega}^{(1)}=\frac{q d}{\hbar \omega} \frac{J_{\mathrm{dark}}\left(V_{\mathrm{dc}}+\hbar \omega / q\right)-\underline{J}_{\mathrm{dark}}\left(V_{\mathrm{dc}}-\hbar \omega / q\right)}{2},
\end{aligned}
$$

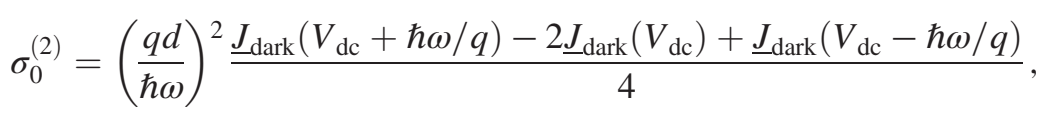



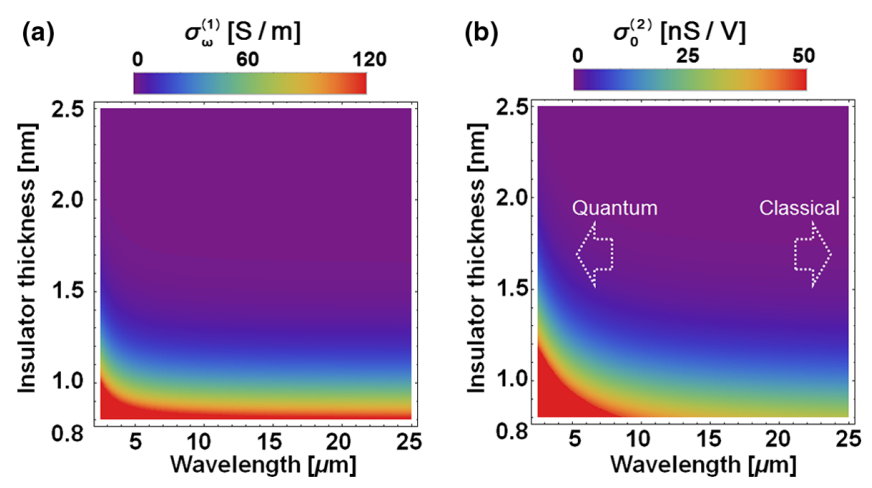

FIG. 2. Contours of (a) linear and (b) nonlinear second-order conductivity induced in a $\mathrm{Ag}-\mathrm{Nb}_{2} \mathrm{O}_{5}-\mathrm{Cu}$ heterojunction, as a function of wavelength and insulator $\left(\mathrm{Nb}_{2} \mathrm{O}_{5}\right)$ thickness.

and $d$ is the thickness of tunnel barrier (insulating layer). It has been experimentally observed and theoretically studied that the power dissipation due to quantum conductance may intrinsically limit the maximum field enhancement achievable in plasmonic nanostructures [1,3,7-11]. Here, we assume that contributions of higherorder harmonics $(m>2)$ are ignorable, since they are significantly attenuated in the proposed geometry.

Here, we consider a HMM structure composed of stacked silver $(\mathrm{Ag})$ and copper $(\mathrm{Cu})$ thin films with thickness $t_{\text {metal }}$, separated by 1 -nm-thick niobium-oxide $\left(\mathrm{Nb}_{2} \mathrm{O}_{5}\right)$ insulating layers [36-39]. Figures 2(a) and 2(b) show contours of $\sigma_{\omega}^{(1)}$ and $\sigma_{0}^{(2)}$ [Eq. (2)], as a function of wavelength and insulator thickness for a $\mathrm{Ag}-\mathrm{Nb}_{2} \mathrm{O}_{5}-\mathrm{Cu}$ heterojunction; here, dc offset $\left(V_{\mathrm{dc}}\right)$ is zero. We find that at long wavelengths (i.e., far-infrared), $\sigma_{\omega}^{(1)}$ and $\sigma_{0}^{(2)}$ approach the classical small-signal limits: $\sigma_{\omega, \text { classical }}^{(1)}=\left.\left(\partial \underline{J}_{\mathrm{dark}} / \partial V_{\mathrm{dc}}\right)\right|_{V_{\mathrm{dc}}=0}$ and $\sigma_{0 \text {, classical }}^{(2)}=\left.(1 / 4)\left(\partial^{2} \underline{J}_{\text {dark }} / \partial V_{\mathrm{dc}}^{2}\right)\right|_{V_{\mathrm{dc}}=0}$, while in the midinfrared regime, $\sigma_{\omega}^{(1)}$ and $\sigma_{0}^{(2)}$ differ significantly from the classical treatment. As seen in Fig. 2, in the shorter wavelength regime $\sigma_{0}^{(2)}$, induced by the photon-assisted tunneling, increases with the energy of incident photon. Such enhancement may benefit the use of midinfrared detection and photovoltaic systems, as the photocurrent is proportional to the nonlinear quantum conductivity $\underline{J}_{\text {photo }} \approx \sigma_{0}^{(2)}\left|E_{\omega}\right|^{2}$. As it can be seen in Fig. 2, $\sigma_{\omega}^{(1)}$ and $\sigma_{0}^{(2)}$ may exist only when a MIM tunneling junction has a nanoor subnanoscale insulating layer. Hence, the nonlinear rectification of infrared light may not be observable in conventional HMMs, which typically have thick dielectric layers. We note that scaling the dielectric thickness of a HMM may find new varieties of nonlinear optical and optoelectronic applications, due to induced linear and nonlinear quantum conductivities [1-13] that may benefit from the large PDOS and tailored dispersion of HMM-based nanostructures.

In the following, we consider a HMM slab that is micromachined with a periodicity of $300-\mathrm{nm}$ and 100-nm-wide air trenches, as shown in Fig. 1(a). A plane wave with transverse-magnetic polarization is incident along the $-\hat{\mathbf{z}}$ direction. The nanotrenched HMM substrate is, in some sense, similar to a periodic array of air-HMM-air waveguides, for which a guided mode propagating along the waveguide axis may be tailored to have a near-zero group velocity, i.e., $v_{g}=\partial \omega / \partial \beta \approx 0$, at certain wavelengths, where $\omega$ is angular frequency and $\beta$ is the modal propagation constant. Based on Maxwell's equations and periodic boundary conditions, the dispersion relation between $\omega$ and $\beta$ can be derived based on the transverse resonance method $[40,41]$ as

$$
\tan \left[k^{\prime} \frac{P-W}{2}\right]-\epsilon_{\perp} \frac{k^{\prime \prime}}{k^{\prime}} \tan \left[k^{\prime \prime} \frac{W}{2}\right]=0,
$$

where $k^{\prime}=\sqrt{k_{0}^{2} \varepsilon_{\perp}-\left(\varepsilon_{\perp} / \varepsilon_{\|}\right) \beta^{2}}$ (i.e., inside the HMM $\left.k^{\prime 2} / \varepsilon_{\perp}+\beta^{2} / \varepsilon_{\|}=k_{0}^{2}\right), k^{\prime \prime}=\sqrt{\beta^{2}-k_{0}^{2}}, k_{0}$ is the free-space wave number, $P$ is the period of unit cells, and $W$ is the width of air slots [see Fig. 3(a)]. Provided that the periodicity of each MIM unit cell is subwavelength $\left(P \ll \lambda_{0}\right)$, all diffraction orders, except for the zeroth mode, are evanescent.

Figure 3(a) shows the dispersion diagram of this HMM structure. In our calculation, we use realistic electronic and optical properties of materials extracted from previous experimental works [42], and consider the dissipative
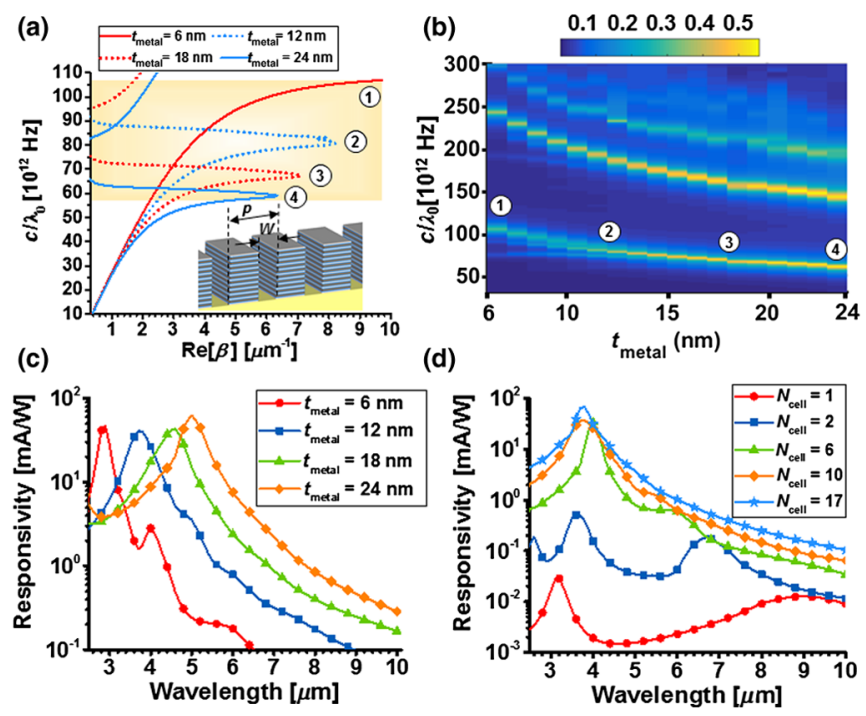

FIG. 3 (a) Dispersion diagram for a waveguide array made by carving a HMM substrate $(P=300 \mathrm{~nm}$ and $W=100 \mathrm{~nm})$. (b) Contours of absorptance for the HMM in (a), varying the metal thickness $\left(t_{\text {metal }}\right)$ and the operating wavelength; labels in (a) and (b) indicate the slow-wave modes and the corresponding absorption peaks. (c) Responsivity spectrum of the HMM in (a), varying the metal thickness (here, the number of MIM unit cells $N_{\text {cell }}=12$ ). (d) is similar to (c), but varying the number of stacked MIM unit cells (here, metal thickness $t_{\text {metal }}=12 \mathrm{~nm}$ ). 
power due to quantum conductivity [Eq. (2a)] [41]. It is seen from Fig. 3(a) that near-zero group velocity can be achieved at critical wavelengths, implying that the incident light is slowed down and trapped inside the lossy and anisotropic HMM region, being absorbed due to plasmon loss. Figure 3(b) shows the associated contour plot of absorptance as a function of metal thickness $t_{\text {metal }}[\mathrm{nm}]$ and wavelength $\lambda[\mu \mathrm{m}]$, calculated by full-wave simulation [43]; here, 12-MIM heterojunctions are assumed and, for simplicity, the effect of the substrate is neglected. We find excellent agreement between the wavelengths of maximum absorption in Fig. 3(b) and the near-zero group velocity points in the dispersion diagram [Fig. 3(a)]. We note that total HMM size is still subwavelength, since the large value of $\operatorname{Re}[\beta]$ suggests that the incident light can be effectively absorbed in a long-wavelength range. Moreover, the absorption spectrum can be readily tailored by varying the volume fraction of metal, which determines the permittivity tensor elements of HMM. In a semiclassical analysis, the (photo) responsivity of this HMM-based structure can be calculated as

$$
\gamma=\frac{\sum_{i=1}^{2 N_{\text {cell }}-1} 2 \int_{W / 2}^{P / 2} \sigma_{0}^{(2)}\left|\left(\mathbf{E}_{\omega}(x) \cdot \hat{\mathbf{z}}\right)\right|^{2} d x}{\frac{\varepsilon_{0} c}{2}\left|\mathbf{E}_{\mathrm{inc}}\right|^{2} P},
$$

where $\mathbf{E}_{\text {inc }}$ is the electric field of the incident light, $\mathbf{E}_{\omega}(x)$ is the electric field inside the MIM gap, $\varepsilon_{0}$ and $c$ are the permittivity and speed of light in free space, and $N_{\text {cell }}$ is the number of unit cells. We note that $\left|\mathbf{E}_{\omega}\right|$ can be largely enhanced compared to $\left|\mathbf{E}_{\text {inc }}\right|$, due to the plasmon coupling across nanogaps, as discussed in Refs. [44-47]. We note that large field enhancement and excellent mode overlap with the nonlocal nonlinear conductivity are both of importance to achieving high responsivity. For the patterned HMM in Fig. 3, the longitudinal component of electric field can be greatly enhanced inside the nanogap, preferentially aligned along the direction of plasmon tunneling [41]. Figure 3(c) shows the calculated responsivity spectrum for different metal thicknesses. It is seen that the peak responsivity can reach a few tens of $\mathrm{mA} / \mathrm{W}$, with a scalable operating wavelength range that is determined by the volume fraction of metals. Figure 3(d) studies the influence of the number of MIM unit cells on responsivity; here, the metal thickness is fixed to $12 \mathrm{~nm}$. As expected, the responsivity is increased by depositing more MIM unit cells. We find that a HMM consisting of a large number of MIM unit cells could have a responsivity up to the $0.1-\mathrm{A} / \mathrm{W}$ level, without any external dc bias. A single-layer MIM device (e.g., flatland metasurface with $N_{\text {cell }}=1$ ), however, shows poor responsivities compared with stereo metamaterials. Interestingly, this HMM-based rectifying device may have the inherent advantage of being angle independent, because the operating wavelength simply depends on the eigenmode propagation inside each subwavelength air-HMM-air waveguide.
Figure 4(a) shows the contour plot of absorptance as a function of operating wavelength and angle of incidence for the same devices in Fig. 3, with $t_{m}=12 \mathrm{~nm}$ and $N_{\text {cell }}=12$ (top panel), and the associated responsivity spectrum for different angles of incidence (bottom panel). From Fig. 4(a), we find omnidirectional absorption at wavelength around $4 \mu \mathrm{m}$ and a high zero-bias responsivity $(\sim 50 \mathrm{~mA} / \mathrm{W})$ at all angles.

The linearly tapered HMM strips, such as the sawtooth geometry reported in Ref. [28], have been used to enhance the bandwidth of operation, based on the superposition of multiple slow-wave modes. However, the gradient shape with a specific tilt angle requires careful control in nanofabrication and could suffer from a lack of device repeatability and uniformity. On the other hand, we know from Figs. 3(a) and 3(b) that the wavelength of slow-light operation can be tuned by changing the volume fraction of metal. We propose here a simple and effective alternative design in Fig. 4(b), which no longer requires tapering the HMM strips. Instead, the superposition of several slowwave modes is achieved by gradually varying the thickness of metal films along the light propagation direction. Figure 4(b) is similar to Fig. 4(a), but for an inhomogeneous-HMM (IH-HMM) rectifying device with $t_{\text {metal }}$ varied from $24 \mathrm{~nm}, 18 \mathrm{~nm}, 12 \mathrm{~nm}$, to $6 \mathrm{~nm}$ (from top to bottom); here, the total number of unit cells is the same as the homogeneous-HMM (H-HMM) device in Fig. 4(a). It is seen that broadband absorption, spanning from 3 to $6 \mu \mathrm{m}$, can be achieved due to the combination of multiple slowwave modes. The peak responsivity of the IH-HMM device is somewhat reduced compared to the H-HMM device with the same number of unit cells, due to the trade-off between bandwidth and maximum absorption. The IH-HMM device may achieve broadband, angle-independent, efficient midinfrared rectification (a zero-bias responsivity $\sim 30 \mathrm{~mA} / \mathrm{W}$ ), with the maximum possible bandwidth highlighted in the dispersion diagram of Fig. 3(a).
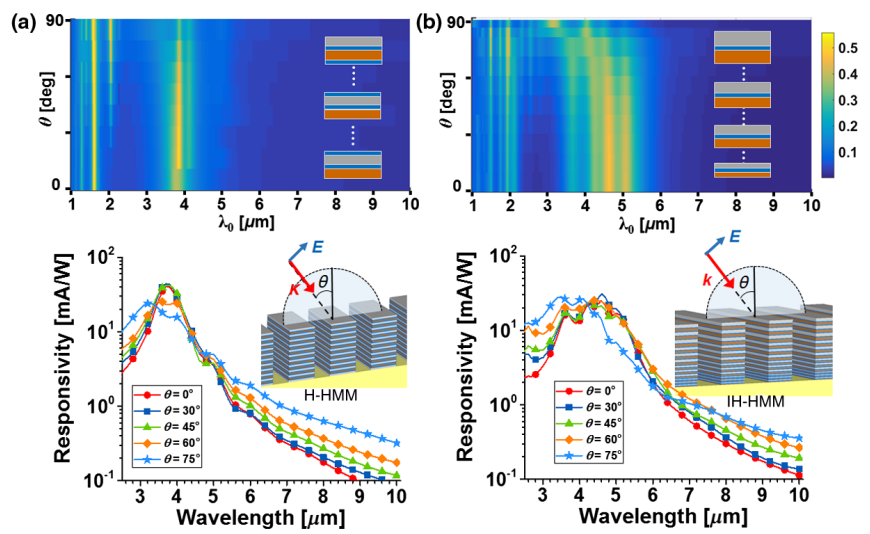

FIG. 4 (a) Contours of absorptance as a function of wavelength and incident angle of infrared for a homogeneous-HMM (H-HMM) device (top), and the corresponding responsivity spectrum (bottom). (b) is similar to (a), but for an inhomogeneous-HMM (IH-HMM) device. 

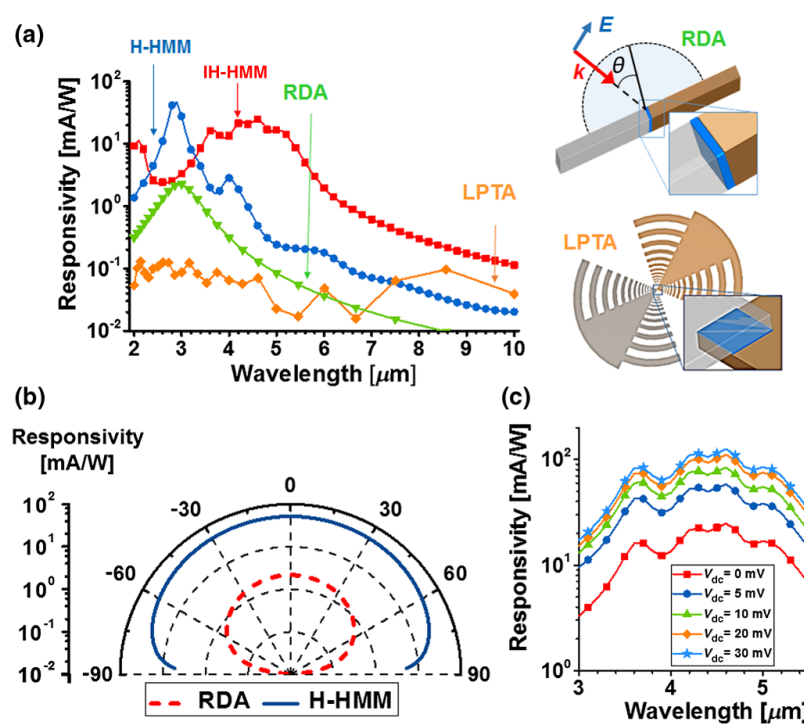

(c)

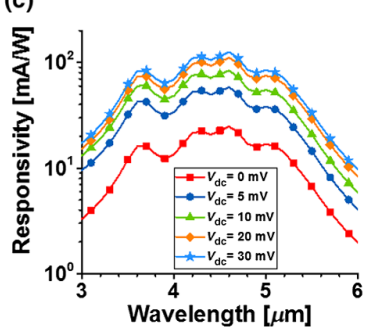

FIG. 5 (a) Responsivity spectrum for different infrared rectifying devices made of the homogeneous-HMM (H-HMM) device, the inhomogeneous-HMM (IH-HMM) device, the resonant dipole antenna (RDA), and the broadband log period tooth antenna (LPTA); the right panel shows geometries of the RDA and LPTA, which are loaded with a MIM diode at their feed points. (b) Angular responses of H-HMM-based and RDA-based devices in a polar plot. (c) Responsivity spectrum of the IH-HMM-based device under different forward-bias voltages.

Next, we compare the performance of H-HMM and IH-HMM devices with conventional infrared nanorectennas [41], which are a broadband log-periodic tooth antenna (LPTA) [48] and a resonant dipole antenna (RDA) loaded with the MIM diode at the feed point. Figure 5(a) shows the responsivity for various infrared rectifying devices consisting of the same $\mathrm{Ag}-\mathrm{Nb}_{2} \mathrm{O}_{5}-\mathrm{Cu}$ tunneling junction and a barrier thickness of $1 \mathrm{~nm}$. The rectenna responsivity is calculated as the ratio of rectified current to the intercepted power (i.e., radiant power density multiplied by the antenna's effective aperture: $D \lambda_{0}^{2} / 4 \pi$, where $D$ is the antenna directivity [48-49]). Here, the MIM diode area is fixed to $100 \mathrm{~nm} \times 100 \mathrm{~nm}$ for LPTA and $30 \mathrm{~nm} \times$ $30 \mathrm{~nm}$ for RDA, which is sufficiently small to avoid a parasitic cutoff frequency. From Fig. 5(a), it is seen that the HMM-based techniques can have much higher responsivities and broader bandwidth compared with lumpedelement-based MIM devices $(0.01-1 \mathrm{~mA} / \mathrm{W})$. The responsivity values obtained for nanorectennas are consistent with previous theoretical works [20-21]. For our nanopatterned HMMs, the improvement in responsivity may be attributed to the broadband and omnidirectional infrared absorption, combined with enhanced nonlinear optical rectification due to the interplay between plasmon coupling and photon-assisted tunneling within the MIM superlattices, making efficient usage of device area and density with massively parallel rectifying junctions. Figure 5(b) compares the angular response for the RDA-based and H-HMM-based devices. It is seen that the HMM-based device is rather insensitive to the angle of illumination, compared with conventional antenna-coupled designs. We note that the LPTA has a poor angular response due to the directive radiation pattern at short wavelengths. Figure 5(c) shows the responsivity of the broadband IH-HMM device in Fig. 4(b) under different forward-bias conditions. It is seen that the responsivity increases with forward biasing, and it can be greater than $0.1 \mathrm{~A} / \mathrm{W}$ over a broad midinfrared range, at a relatively small bias voltage.

Finally, we believe that the described HMM-based devices may be achievable within currently available nanofabrication technologies. The metal and transition-metal oxide thin films can be fabricated using atomic layer deposition [36-38]. The waveguide array can be fabricated by advanced lithographic methods, including electron-beam lithography, laser-beam direct writing, or focused-ion-beam milling [28-31]. The relatively mature interconnect technology in high-density integrated circuits [50] may connect different metal layers to enable the efficient collection of photocurrents.

In conclusion, we have put forward the concept and realistic designs of efficient, angle-independent, broadband and wavelength-selective infrared rectification, based on the slow-wave HMM substrate with a broad singularity in the photonic density of states. When a HMM is formed by dissimilar metals and ultrathin atomic-layer-deposited dielectric layers, the photon-assisted tunneling as a quantum mechanical effect takes place, introducing a quantum conductance in MIM superlattices. We have theoretically demonstrated that the second-order quantum conductivity induced in the HMM-based slow-wave medium, when combined with concentrated optical fields in coupled plasmonic nanojunctions, can provide a $\sim 0.1 \mathrm{~A} / \mathrm{W}$-level midinfrared responsivity at zero bias. This HMM-based infrared device also enables an ultrafast response and it does not require any cryogenic cooling, as typical properties of MIM devices. We envision that this nonlinear rectification method may be applied to several applications of interest at midinfrared, including detection, imaging, sensing, communication, and energy harvesting.

[1] J. Zuloaga, E. Prodan, and P. Nordlander, Quantum description of the plasmon resonances of a nanoparticle dimer, Nano Lett. 9, 887 (2009).

[2] M. L. Brongersma, N. J. Halas, and P. Nordlander, Plasmon-induced hot carrier science and technology, Nat. Nanotechnol. 10, 25 (2015).

[3] D. C. Marinica, A. K. Kazansky, P. Nordlander, J. Aizpurua, and A. G. Borisov, Quantum plasmonics: Nonlinear effects in the field enhancement of a plasmonic nanoparticle dimer, Nano Lett. 12, 1333 (2012).

[4] A. Stolz, J. Berthelot, M.-M. Menemanteuil, G. C. des Francs, L. Markey, V. Meunier, and A. Bouhelier, Nonlinear 
photon-assisted tunneling transport in optical gap antennas, Nano Lett. 14, 2330 (2014).

[5] G. Hajisalem, M. S. Nezami, and R. Gordon, Probing the quantum tunneling limit of plasmonic enhancement by third harmonic generation, Nano Lett. 14, 6651 (2014).

[6] J. Lee, M. Tymchenko, C. Argyropoulos, P. Y. Chen, F. Lu, F. Demmerle, G. Boehm, M. C. Amann, A. Alù, and M. A. Belkin, Giant nonlinear response from plasmonic metasurfaces coupled to intersubband transitions, Nature (London) 511, 65 (2014).

[7] J. W. Haus, D. de Ceglia, M. A. Vincenti, and M. Scalora, Quantum conductivity for metal-insulator-metal nanostructures, J. Opt. Soc. Am. B 31, 259 (2014).

[8] J. W. Haus, D. de Ceglia, M. A. Vincenti, and M. Scalora, Nonlinear quantum tunneling effects in nanoplasmonic environments: Two-photon absorption and harmonic generation, J. Opt. Soc. Am. B 31, A13 (2014).

[9] P. Y. Chen, K. Q. Le, and A Alù, Nonlinear nanocircuitry based on quantum tunneling effects, MRS Commun. 5, 565 (2015).

[10] M. Farhat, M. M. C. Cheng, K. Q. Le, and P. Y. Chen, Nanoantenna harmonic sensor: Theoretical analysis of contactless detection of molecules with light, Nanotechnology 26, 415201 (2015).

[11] P. Y. Chen and M. Farhat, Modulatable optical radiators and metasurfaces based on quantum nanoantennas, Phys. Rev. B 91, 035426 (2015).

[12] P. K. Tien and J. P. Gordon, Multiphoton process observed in the interaction of microwave fields with the tunneling between superconductor films, Phys. Rev. 129, 647 (1963).

[13] J. R. Tucker, Quantum limited detection in tunnel junction mixers, IEEE J. Quantum Electron. 15, 1234 (1979).

[14] S. Grover and G. Moddel, Applicability of metal/insulator/ metal (MIM) diodes to solar rectennas, IEEE J. Photovoltaics 1, 78 (2011).

[15] G. Moddel and S. Grover, Rectenna Solar Cells (Springer, Boulder, CO, 2013).

[16] S. J. Byrnes, R. Blanchard, and F. Capasso, Harvesting renewable energy from Earth's mid-infrared emissions, Proc. Natl. Acad. Sci. U.S.A. 111, 3927 (2014).

[17] A. Krier, Mid-infrared Semiconductor Optoelectronics (Springer, Lancaster, UK, 2006).

[18] J. A. Hagerty, F. B. Helmbrecht, W. H. McCalpin, R. Zane, and Z.B. Popovic, Recycling ambient microwave energy with broad-band rectenna arrays, IEEE Trans. Microwave Theory Tech. 52, 1014 (2004).

[19] The typical response time for electron tunneling across a MIM junction is approximately given by the traversal time $\tau \approx \hbar / \sqrt{E_{F} \Phi}$ where $\hbar$ is the reduced Planck's constant, $E_{F}$ is the Fermi energy, and $\Phi$ is the work function of metal. S. M. Faris, T. K. Gustafson, and J. C. Wiesneret, Detection of optical and infrared radiation with DC-biased electrontunneling metal-barrier-metal diodes, IEEE J. Quantum Electron. 9, 737 (1973).

[20] S. Grover, O. Dmitriyeva, M. J. Estes, and G. Moddel, Traveling-wave metal/insulator/metal diodes for improved infrared bandwidth and efficiency of antenna-coupled rectifiers, IEEE Trans. Nanotechnol. 9, 716 (2010).

[21] E. Briones, J. Alda, and F. J. Gonzalez, Conversion efficiency of broad-band rectennas for solar energy harvesting applications, Opt. Express 21, A412 (2013).
[22] D. R. Ward, F. Huser, F. Pauly, J. C. Cuevas, and D. Natelson, Optical rectification and field enhancement in a plasmonic nanogap, Nat. Nanotechnol. 5, 732 (2010).

[23] Z. Liu, H. Lee, Y. Xiong, C. Sun, and X. Zhang, Far-field optical hyperlens magnifying sub-diffraction-limited objects, Science 315, 1686 (2007).

[24] Z. Jacob, J. Y. Kim, G. V. Naik, A. Boltasseva, E. E. Narimanov, and V. M. Shalaev, Engineering photonic density of states using metamaterials, Appl. Phys. B 100, 215 (2010).

[25] A. Poddubny, I. Iorsh, P. Belov, and Y. Kivshar, Hyperbolic metamaterials, Nat. Photonics 7, 948 (2013).

[26] M. Y. Shalaginov, V. V. Vorobyov, J. Liu, M. Ferrera, A. V. Akimov, A. Lagutchev, A. N. Smolyaninov, V. V. Klimov, J. Irudayaraj, A. V. Kildishev, A. Boltasseva, and V. M. Shalaev, Enhancement of single-photon emission from nitrogen-vacancy centers with $\mathrm{TiN} /(\mathrm{Al}, \mathrm{Sc}) \mathrm{N}$ hyperbolic metamaterial, Laser Photonics Rev. 9, 120 (2015).

[27] W. D. Newman, C. L. Cortes, and Z. Jacob, Enhanced and directional single-photon emission in hyperbolic metamaterials, J. Opt. Soc. Am. B 30, 766 (2013).

[28] Y. Cui, K. H. Fung, J. Xu, H. Ma, Y. Jin, S. He, and N. X. Fang, Ultrabroadband light absorption by a sawtooth anisotropic metamaterial slab, Nano Lett. 12, 1443 (2012).

[29] H. Hu, D. Ji, X. Zeng, K. Liu, and Q. Gan, Rainbow trapping in hyperbolic metamaterial waveguide, Sci. Rep. 3, 1249 (2013).

[30] D. Lu, J. J. Kan, E. E. Fullerton, and Z. Liu, Enhancing spontaneous emission rates of molecules using nanopatterned multilayer hyperbolic metamaterials, Nat. Nanotechnol. 9, 48 (2014).

[31] J. Zhou, A. F. Kaplan, L. Chen, and L. J. Guo, Experiment and theory of the broadband absorption by a tapered hyperbolic metamaterial array, ACS Photonics 1, 618 (2014).

[32] K. S. Giboney, M. J. W. Rodwell, and J.E. Bowers, Traveling-wave photodetector theory, IEEE Trans. Microwave Theory Tech. 45, 1310 (1997).

[33] J. G. Simmons, Generalized thermal $J-V$ characteristic for the electric tunnel effect, J. Appl. Phys. 35, 2655 (1964).

[34] J. G. Simmons, Generalized formula for the electric tunnel effect between similar electrodes separated by a thin insulating film, J. Appl. Phys. 34, 1793 (1963).

[35] J. G. Simmons, Electric tunnel effect between dissimilar electrodes separated by a thin insulating film, J. Appl. Phys. 34, 2581 (1963).

[36] N. Alimardani and J.F. Conley, Jr., Enhancing metalinsulator-insulator-metal tunnel diodes via defect enhanced direct tunneling, Appl. Phys. Lett. 105, 082902 (2014).

[37] N. Alimardani, J. M. McGlone, J. F. Wager, and J. F. Conley, Jr., Conduction processes in metal-insulator-metal diodes with $\mathrm{Ta}_{2} \mathrm{O}_{5}$ and $\mathrm{Nb}_{2} \mathrm{O}_{5}$ insulators deposited by atomic layer deposition, J. Vac. Sci. Technol. A 32, 01A122 (2014).

[38] E. W. Cowell, N. Alimardani, C. C. Knutson, J. F. Conley, D. A. Keszler, B. J. Gibbons, and J. F. Wager, Advancing MIM electronics: Amorphous metal electrodes, Adv. Mater. 23, 74 (2011).

[39] S. Grover and G. Moddel, Engineering the current-voltage characteristics of metal-insulator-metal diodes using double-insulator tunnel barriers, Solid State Electron. 67, 94 (2012). 
[40] N. Marcuvitz, Waveguide Handbook (McGraw-Hill, New York, 1951).

[41] See Supplemental Material at http://link.aps.org/ supplemental/10.1103/PhysRevApplied.5.041001 for more details on the generalized formula for the electric tunnel effect in MIM system, eigenmode analysis, and field distributions of HMM-waveguide arrays and nanorectenna designs.

[42] P. B. Johnson and R. W. Christy, Optical constants of the noble metals, Phys. Rev. B 6, 4370 (1972); P. R. West, S. Ishii, G. V. Naik, N. K. Emani, V. M. Shalaev, and A. Boltasseva, Searching for better plasmonic materials, Laser Photonics Rev. 4, 795 (2010); N. Alimardani, S. W. King, B. L. French, C. Tan, B. P. Lampert, and J. F. Conley, Jr., Investigation of the impact of insulator material on the performance of dissimilar electrode metal-insulator-metal diodes, J. Appl. Phys. 116, 024508 (2014). Below the onset of metal's interband transition, the relative permittivity of metal following the Drude-type dispersion model: $\varepsilon_{m}=\varepsilon_{\infty}-\omega_{p}^{2} /[\omega(\omega+i \gamma)]$, where the empirical parameters for $\mathrm{Cu}$ are $\omega_{p} / 2 \pi=2100 \mathrm{THz}, \gamma / 2 \pi=16.9 \mathrm{THz}$ and $\varepsilon_{\infty}=6.7$, and those for $\mathrm{Ag}$ are $\omega_{p} / 2 \pi=2175 \mathrm{THz}$, $\gamma / 2 \pi=4.35 \mathrm{THz}$ and $\varepsilon_{\infty}=5$. The work functions of metals are $\Phi_{1}=4.26 \mathrm{eV}(\mathrm{Ag})$ and $\Phi_{2}=4.7 \mathrm{eV}(\mathrm{Cu})$. The electron affinity $\chi$, dielectric constant $K$, and relative permittivity (in the midinfrared range) of $\mathrm{Nb}_{2} \mathrm{O}_{5}$ are 4 , 20 , and $5.5 \mathrm{eV}$, respectively. For simplicity, we ignore quantum-size effects of metal, since the minimum thickness of metal film is slightly above the critical dimension (e.g., 3 nm [L. A. Kuzik, V. A. Yakovlev, F. A. Pudonin, and G. Mattei, Surf. Sci. 361, 882 (1996)]). For extremely thin metals, the quantum film experiences a confinement effect and a finite density of states must be taken into consideration, which will vary the effective permittivity.

[43] CST Microwave Studio, http://www.cst.com.

[44] A. Alù and N. Engheta, Tuning the scattering response of optical nanoantennas with nanocircuit loads, Nat. Photonics 2, 307 (2008).

[45] P. Y. Chen and A. Alù, Optical nanoantenna arrays loaded with nonlinear materials, Phys. Rev. B 82, 235405 (2010).

[46] P. Y. Chen and A. Alù, Subwavelength imaging using phaseconjugating nonlinear nanoantenna arrays, Nano Lett. 11, 5514 (2011).

[47] P. Y. Chen, M. Farhat, and A. Alù, Bistable and SelfTunable Negative-Index Metamaterial at Optical Frequencies, Phys. Rev. Lett. 106, 105503 (2011).

[48] C. A. Balanis, Antenna Theory: Analysis and Design (John Wiley \& Sons, Hoboken, NJ, 2005).

[49] P. Y. Chen, C. Argyropoulos, and A. Alù, Plasmonic Optical Nanoantennas, in Modern Plasmonics (Elsevier, Chicago, 2014).

[50] M.S. Bakir and J.D. Meindl, Integrated Interconnect Technologies for 3D Nanoelectronic Systems (Artech House, Boston, MA, 2008). 